\title{
Problèmes hivernaux liés à la neige et au vent
}

Le transport de la neige par le vent est un phénomène physique souvent observé pendant la saison hivernale. Il a de nombreuses conséquences négatives. En haute montagne, il conduit à la mise en place de suraccumulations de neige instable alors que dans le même temps des portions de pistes de ski voient leur neige emportée par le vent. Enfin, dans d'autres cas (cols, plateaux), on rencontrera de graves problèmes de viabilité hivernale qui se traduisent par la formation de congères et par des pertes de visibilité importantes.

C'est pour ces raisons que, dans le cadre du contrat Etat-Région du $X^{\mathfrak{c}}$ plan, a été financé le programme d'étude proposé par trois organismes : le CEMAGREF (division Nivologie), METEO-FRANCE (CEN, Centre d'études de la neige) et l'Université Joseph Fourier (LAMA, Laboratoire d'études de la montagne alpine). A ce groupe, s'est jointe l'Ecole polytechnique fédérale de Lausanne (EPFL), qui est liée par une convention de collaboration avec le CEMAGREF pour l'étude du transport de la neige par le vent.

Ce programme a tout d'abord consisté en l'équipement d'un laboratoire d'altitude situé à proximité du domaine skiable de l'Alpe d'Huez. L'aide logistique importante apportée par cette station a été une raison du choix de ce site, qui présente par ailleurs des caractéristiques physiques intéressantes.

Nous en sommes maintenant aux premiers résultats et dans les lignes suivantes seront présentés certains apports ou méthodologies des trois équipes:

- tout d'abord ceux du CEMAGREF/EPFL, avec de nombreuses techniques de visualisation des particules en mouvement (plan laser, vidéo, photo...) ou de répartition (stéréophotogrammétrie);

- ensuite ceux du CEN, avec l'essai de validation du capteur hauteur de neige pour repérer les épisodes de transport; et la détermination de seuils de vent propice au transport fonction du type de neige de surface; - enfin ceux du LAMA, avec la campagne de mesures radiométriques (réflectance) de la neige, dont le but est d'étudier les relations entre paramètres de surface du manteau neigeux et les caractéristiques radiométriques pour une utilisation en télédétection.

Transport de la neige par le vent en montagne Mesure sur site et en laboratoire, première modélisation

Thierry CASTElle, Alain ClapPier - Laboratoire des Systèmes énergétiques, Ecole polytechnique fédérale de Lausanne

Martine Roussel - Division Nivologie, CEMAGREF, Saint-Martin d'Hères

La prévision des effets du transport de la neige par le vent en montagne débute. Elle nécessite de créer et d'adapter des techniques de modélisation numérique et expérimentale. Ces modèles sont calés et validés par des mesures in situ en haute montagne et par des expériences de trajectographie en soufflerie.

A partir d'une recherche bibliographique et de nos premières analyses du phénomène, nous avons programmé la base d'un modèle simplifié de transport de la neige par saltation.
Au cours de cette première année du projet, l'effort a été porté sur le développement de techniques de mesure in situ et en soufflerie. Sur le terrain, nous avons construit et équipé en collaboration avec le CEN un laboratoire d'altitude (Alpe d'Huez 2720 m). Nous mesurons et enregistrons l'évolution des conditions nivo-météorologiques au cours de l'hiver. Sur ce site nous menons aussi des campagnes d'observation des différents phénomènes de transport de la neige. Ainsi, nous avons mis au point plusieurs capteurs de flux de neige et des systèmes de description du comportement des particules de

LA HOUILLE BLANCHE/N 5-1991 
neige en saltation. En particulier, nous avons adapté aux conditions extérieures la technique de visualisation laser par nappe dynamique. La technique de stéréophotogrammétrie terrestre permet de suivre l'évolution des dépôts de neige à grande échelle en fonction des conditions météorologiques.

En soufflerie, un ensemble capteur optique-logiciel d'acquisition permet la mesure des hauteurs de dépôts sur les maquettes. Une nappe laser dynamique associée à un logiciel d'acquisition et de traitement des images permet de calculer la vitesse et la concentration des particules dans l'écoulement.

\section{Introduction}

Neige et vent : des conséquences connues, des mécanismes mal connus

Congères, avalanches de plaque, pistes de ski déneigées par le vent, autant de soucis pour le gestionnaire ou l'usager de sites enneigés. Cependant leurs mécanismes sont encore mal connus.

La prévision des accumulations, ou érosions, passent par une description puis une modélisation des phénomènes. Nous développons des outils d'étude et de modélisation du transport de la neige en montagne. L'objectif final de ces modèles est de simuler, à partir d'une situation météorologique donnée ou supposée, la position des zones d'érosion et d'accumulation de neige sur un site donné (route, région montagneuse).

Un modèle se développe à partir d'hypothèses de départ, d'explications de mécanismes, puis se teste par comparaison de simulations avec des cas réels (calage). Il faut donc avant tout se doter de moyens d'observations et de mesures sur le terrain ou sur maquettes (soufflerie).

Nous présenterons ici les hypothèses que nous voulons vérifier, l'instrumentation spécifique dont nous nous sommes doté et le type de modèle que nous développons depuis un an.

\section{Jusqu'à présent, peu d'études en montagne}

L'étude du transport de neige par le vent est relativement récente (début des années 60) et s'inspire des travaux réalisés sur le sable en 1941, par BAGNOLD. Celui-ci a décrit le transport de particules lourdes dans l'air et défini la notion de saltation: sous l'action du vent et de la gravité les particules de neige adoptent une trajectoire parabolique dissymétrique. Avec l'introduction des théories relatives aux couches limites turbulentes, les travaux d'OWEN (1964) ont permis de mieux comprendre les mécanismes d'érosion et de saltation, ceci dans des conditions de laboratoire. Les mesures in situ du phénomène de saltation ont été poussées très loin par MELLOR (1964), RADOK (1968), TABLER
(1973, 1980) et SchmidT (1980). Mais ces travaux concernent des régions au relief peu prononcé : régions polaires et grands plateaux continentaux. Dans ces conditions $80 \%$ du transport s'effectue par saltation.

Curieusement, très peu d'observations correspondent à la topographie accidentée des Alpes. Dans le cas des montagnes, les travaux de FÖHN (1980), HerTig (1987) et MEISTER (1989) montrent que la topographie et l'altitude jouent un rôle important dans la distribution spatiale des dépôts (influence du sillage des crêtes lors de la chute de neige et de la qualité de la neige sur les accumulations). Mais certains phénomènes et non des moindres (congères, plaques à vent) sont principalement dus au frittage des particules par saltation.

D'autre part, les travaux très récents de $\mathrm{Ph}$. Yverniaux (1990) et de F.S. TARDU (1988) sur l'effet des bouffées turbulentes et sur l'éjection des particules incitent à reconsidérer le mécanisme de saltation.

Comme on le voit, l'approche du problème est complexe car elle nécessite des connaissances dans les domaines de la turbulence, du transport des particules, de la nivologie et de la météorologie. Par conséquent, seule une approche pluridisciplinaire combinant les mesures in situ, les essais en laboratoire et les modèles numériques a des chances d'aboutir.

\section{Des organismes unissent leurs moyens}

Par une collaboration entre plusieurs instituts permettant de bénéficier de l'expérience acquise dans de nombreux domaines (nivologie, laboratoire, numérique) nous menons une recherche pluridisciplinaire:

EPFL : Ecole polytechnique fédérale de Lausanne : coordination scientifique, modélisation numérique, campagne de mesures.

CEMAGREF : Nivologie : soutien logistique, développement de l'instrumentation, campagnes de mesure, soufflerie.

CEN : (Centre d'étude de la neige de Météo-France): enregistrement des conditions nivo-météorologiques et recherche spécifique.

\section{... sur un site d'étude commun...}

Un laboratoire d'altitude a été installé en 1989 au col du Lac Blanc $(2720 \mathrm{~m})$ dans le massif des petites Rousses (Oisans). Ce site a été choisi pour son caractère haute montagne (conditions climatiques difficiles) et pour la canalisation des vents très marquée (Axe Nord-Sud).

Le laboratoire est constitué par un chalet de $16 \mathrm{~m}^{2}$ doté d'une alimentation secteur, du chauffage et du téléphone. L'accès est facilité par la présence du téléphérique de Vaujany (SATA) situé à $300 \mathrm{~m} \mathrm{du} \mathrm{col} \mathrm{(fig.} \mathrm{1).}$

\section{... pour étudier}

Dans ce cadre, nous avons entrepris des expériences destinées à : 


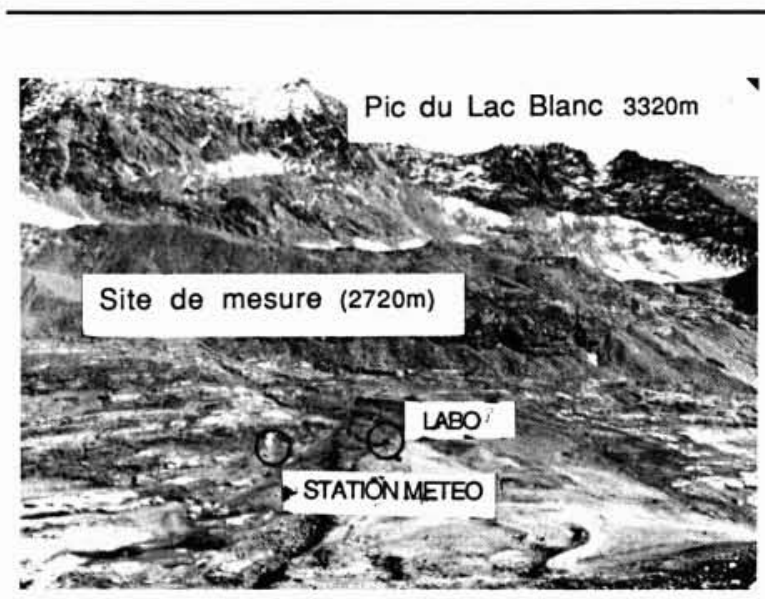

1. Site du col du Lac Blanc.

- déterminer les particularités des transports de neige en zone alpine

- vérifier des hypothèses sur le mécanisme de saltation - accumuler les données permettant le calage et une validation du modèle à partir des situations réelles.

\section{Instrumentation}

\subsection{Le choix}

\section{Mesurer quoi?}

Les mesures en soufflerie ou en montagne devront montrer :

Quelle est l'importance relative des différents mécanismes dont le vocabulaire est à définir, en fonction des conditions nivo-météorologiques et topographiques?

Quelles sont les conséquences de chacun des phénomènes sur la répartition et la forme des dépôts observés?

$\mathrm{Si}$ les notions de saturation de l'écoulement et de capacité limite de transport mesurées en terrains plats (expérience de Takeuchi (1980)) sont valables en haute montagne ?

Si les bouffées turbulentes observées dans les études de mise en suspension des particules de sable dans l'eau existent-elles dans le cas de l'air et de la neige ?

Quelles sont les limites des études en soufflerie pour l'étude du transport de neige en milieu naturel?

Quelle est la différence entre les résultats des modèles et la réalité ?

Répondre à ces questions est un travail de longue haleine, que nous avons débuté il y a un an. Nous présentons la démarche qui a guidé nos choix, mais nous vous épargnerons un inventaire exhaustif et détaillé des expériences.

Choisir les capteurs nécessaires... et suffisants

Nous avons développé l'instrumentation en nous imposant quelques principes :
- documenter les conditions nivo-météorologiques avant et pendant une campagne de mesure le plus complètement possible; cela augmentera la qualité et la fiabilité des interprétations ultérieures ;

- utiliser des moyens de mesure similaires en soufflerie et sur le terrain pour faciliter les comparaisons;

- utiliser des capteurs du commerce chaque fois que possible afin de réduire les temps de développement et augmenter la fiabilité ;

- veiller à la complémentarité des méthodes employées, et varier les techniques (mécanique, optoélectronique, imagerie).

\section{... en multipliant les points de vue}

Une grille d'analyse à trois dimensions a permis de limiter les redondances et les vides dans les domaines couverts par nos capteurs (fig. 2 et 3 ). Nous distinguons: les outils décrivant le comportement de la particule (chaque particule est considérée individuellement) de ceux décrivant les caractéristiques du flux de particules (la neige soufflée est alors considérée comme un fluide continu).

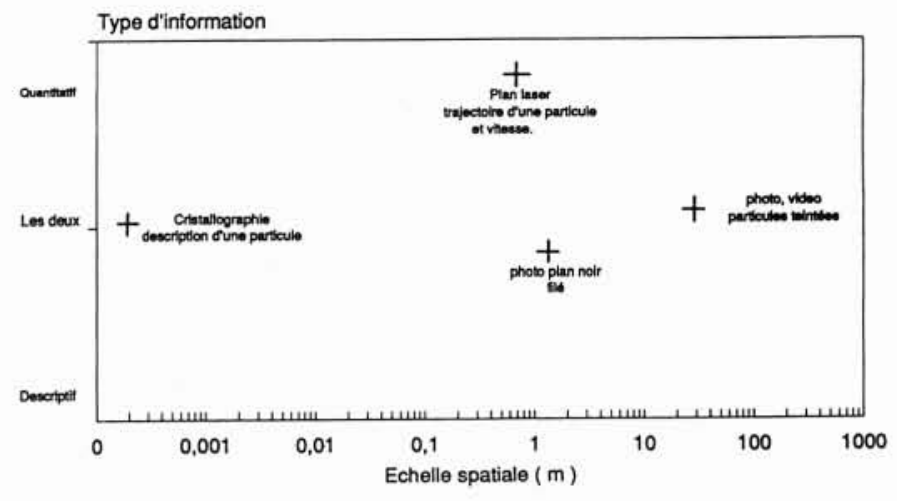

2. Instrumentation pour l'étude du transport de neige à l'échelle de la particule.

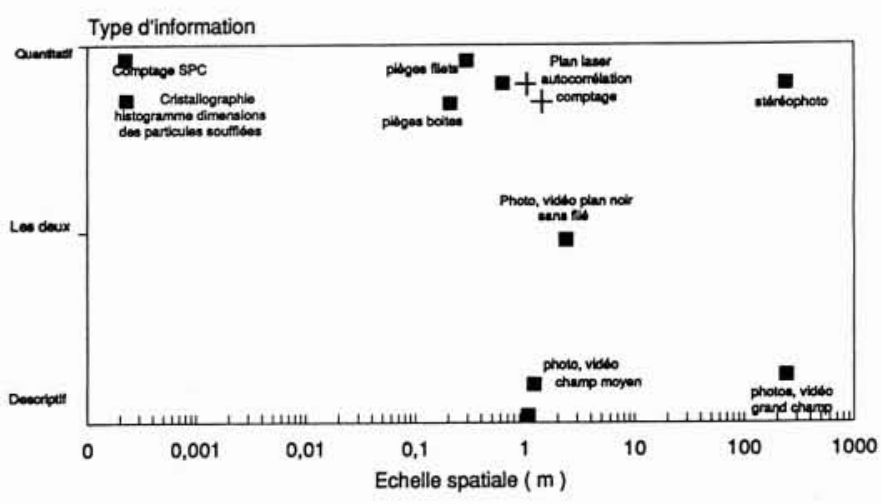

3. Instrumentation pour l'étude du transport de neige à l'échelle d'un flux de particules. 
A cette classification se superpose la notion d'échelle :

- échelle spatiale, du $1 / 10$ de $\mathrm{mm}$ à la centaine de mètre ; - échelle temporelle de la durée de l'observation ou de l'intervalle entre les observations, variable du 1/100 de seconde à la semaine.

et le degré d'objectivité des informations obtenues :

- qualitatives (aide à la description, ou étalonnage impossible);

- quantitatives.

Afin de ne pas ennuyer le lecteur par une énumération fastidieuse, nous avons représenté sur ces tableaux toutes les expériences pour lesquelles nous sommes équipés, en les faisant apparaître dans l'espace défini ci-dessus (seule l'échelle temporelle n'apparaît pas). La diversité de nos points de vue y apparaît clairement.

\subsection{Le développement}

Nous résumons maintenant les principales caractéristiques de nos outils par famille technologique, après avoir décrit nos moyens de documentation des essais.

\subsubsection{Documenter le contexte des expériences}

Quel expérimentateur ne s'est jamais retrouvé au bureau, avec des notes ou enregistrements inutilisables faute d'avoir noté correctement tous les paramètres? Fâcheuse situation lorsque les conditions ne sont pas reproductibles à volonté ou les expériences trop lourdes à organiser.

Les mesures lors des épisodes de transport ne sont donc exploitables que si le contexte nivo-météorologique a été bien enregistré lors de la mesure.

Pour ceci nous disposons :

1. D'une station météorologique automatique installée par le Centre d'Etude de la Neige. Cette station enregistre toute la saison d'hiver les conditions locales au pas de temps horaire.

D'une part les données sont affichées en temps réel sur un écran dans le chalet, ce qui permet aux opérateurs de choisir les moments intéressants auxquels faire les essais pendant une campagne de mesure (en particulier vitesse et direction du vent).

D'autre part, elles sont utilisables en temps différé pour interpréter par exemple les mesures à temps d'intégration long (rose des vents entre deux dates de relevé de pièges à neige ou deux prises de vue stéréoscopique). De plus, les caractéristiques cristallographiques de la neige de surface sont régulièrement relevées au cours de la saison.

2. D'enregistrements de courte durée (quelques minutes) lors des campagnes de mesure.

Dans ce cas, on contrôle et enregistre les conditions de vent (vitesse et direction) exactement au point où est faite l'expérience (visualisation, profil de flux par piégeage...) : un logiciel affiche ces paramètres en temps réel puis les archives si l'opérateur est satisfait : vent stationnaire suffisamment long, vitesse moyenne intéressante ou autre critère adapté à l'étude. De plus, on décrit la nature de la neige transportée par prise d'échantillons et analyse cristallographique dans la couche en saltation.

\subsubsection{Les capteurs}

- Pièges mécaniques : à la chasse du flocon!

Ce sont des "pièges à neige ", qui perturbent peu l'écoulement d'air mais dans lesquels la neige se dépose.

Par principe, ce sont des capteurs de flux, leur temps d'intégration (durée d'exposition au vent) est aisément variable (compte tenu de leur capacité et des conditions météo). Leur mise en œuvre est simple et leur résistance aux conditions météo est excellente. Par contre la pesée n'est pas automatisable.

Nous disposons de :

-2 profils constitués de 6 boîtes prismatiques (fig. 4), une dirigée vers le Nord, l'autre vers le Sud, de plusieurs jours de capacité de stockage (orifice de $1 \mathrm{~cm}^{2}$ );

-4 perches de 4 mètres de haut équipées de 8 filets (fig. 4) en toile fine de nylon de $30 \mathrm{~cm}^{2}$ d'ouverture, permettant des mesures de quelques minutes à une heure. Plus précis que les boîtes, ils sont utilisés lors des campagnes de mesure pour réaliser des profils de concentration. Signalons qu'un essai en soufflerie a permis de vérifier le peu de perturbation de l'écoulement imputable au filet. Leur coût est très faible.

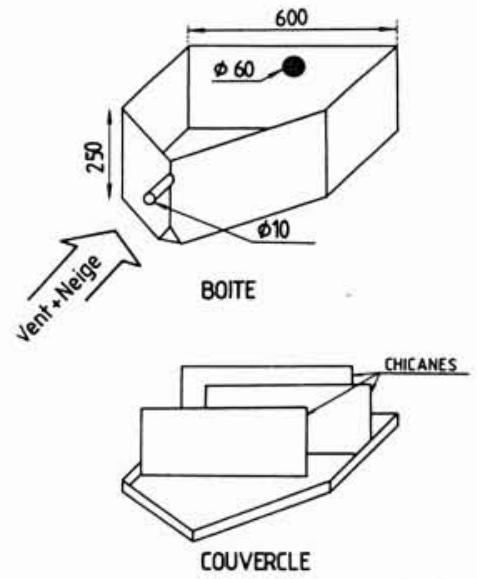

BOITE PRISMATIQUE

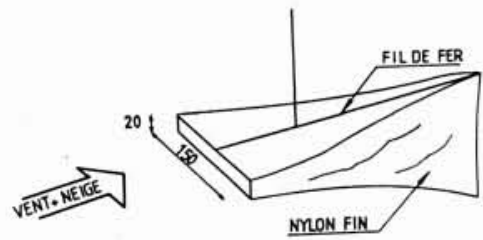

FILET A NEIGE

4. Boite prismatique et filet nylon. 


\section{- Capteurs optoélectroniques, ou la détection sans contact}

Tous les capteurs utilisent un même principe de base : un émetteur de lumière, un récepteur de lumière et une optique. Soit l'objet à détecter s'interpose entre l'émetteur et le récepteur, et la quantité de lumière détectée diminue : il s'agit alors d'une barrière optique, comme on en trouve pour les sécurités de portes d'ascenseur. Soit l'émetteur et le récepteur sont côte à côte, foyers optiques confondus, et alors un objet clair et diffusant placé en ce point renvoie la lumière de l'émetteur vers le récepteur; il s'agit d'un dispositif à réflexion directe; c'est sur ce principe que fonctionnent les lecteurs de code-barre. Certains types de détecteurs permettent de mesurer des distances sans contact.

La mesure est ponctuelle dans l'espace, mais peut être à grande résolution temporelle et continue dans le temps.

Nous utilisons :

- Détecteur automatique de transport : Nous avons acheté un SPC (« snow particule counter " Schmidt (1982)) qui est une barrière optique de section de $3 \mathrm{~mm} \times 0,8 \mathrm{~mm}$, longueur $25 \mathrm{~mm}$. Il s'est avéré trop rudimentaire pour que l'on puisse détecter les plus petites des particules de neige transportées (ce qui ne permettrait pas un comptage précis). Cependant il détecte les particules d'un millimètre de diamètre; nous avons créé un signal du type: «j'ai vu au moins une particule depuis la dernière lecture ». Nous lisons le signal toutes les 20 secondes grâce à la centrale météo automatique. La stabilité dans le temps de ce signal permet de déterminer si le transport est intermittent ou non. Si le capteur n'est pas enfoui sous une couche de neige... il aidera à l'interprétation des mesures de hauteur faites par le capteur à ultrasons. Il est trop encombrant pour une utilisation en soufflerie.

- Profils de concentration en fonction de la hauteur par rapport au sol: Il s'agit d'installer sur un profil de hauteur plusieurs capteurs, compacts, fiables et bon marché, sensibles aux plus petites particules. Nous procéderons cet hiver à l'essai de la partie optique des "lecteurs de code-barre" standards (Hewlett-Packard). Leurs zones de détection est beaucoup plus réduite que celle du SPC $(0,2 \mathrm{~mm} \times$ $0,2 \mathrm{~mm}$, longueur $1,2 \mathrm{~mm}$ ). Le contraste est donc meilleur pour les petites particules. Compacts (un cylindre de $6 \mathrm{~mm}$ de diamètre) ils perturbent très peu l'écoulement, on peut donc les utiliser en soufflerie.

Dans un premier temps, on comptera les particules " vues" par intervalle de temps.

Remarque : La conversion du comptage en flux massique est délicate. Gubler propose une technique; il faut faire l'approximation que les particules sont sphériques, ce que les microphotographies semblent contredire pour le moment !

- Mesure sans contact des hauteurs de dépôt de particules en soufflerie: Un capteur de distance sans contact ("Voyant" de 6 à $14 \mathrm{~cm}$ sous lui) est déplacé selon une grille régulière au-dessus des congères obtenues en soufflerie. Une chaîne de mesure sur ordinateur enregistre les distances et restitue une cartographie tridimensionnelle de la congère.

\subsubsection{L'imagerie}

Voir blanc sur blanc!

Des particules de neige se déplaçant sur un champ de neige : blanc sur blanc! Un cauchemar de photographe. Or les techniques d'imagerie sont les seules qui permettent d'enregistrer ce qui se passe dans une zone, et non pas ponctuellement comme les techniques exposées plus haut. Nous avons donc cherché des artifices (accessoires, conditions de prise de vue, éclairage artificiel) pour augmenter le contraste, et faciliter l'interprétation de l'information. Celle-ci est faite soit par la vision humaine, soit par traitement informatisé.

Photo ou vidéo, quand il faut choisir...

La vidéo a beaucoup d'attraits : 25 images par seconde de plusieurs minutes d'affilé, contrôle immédiat de la qualité de l'enregistrement, capteurs CCD sensibles et sans déformation au cours du temps... Cela ne doit pas masquer une résolution spatiale très faible: 500 points sur 500 points pour décrire tout le champ de vision (enregistrement Super VHS, c'est-à-dire le plus performant des systèmes grand public actuels). Autrement dit, si on veut mesurer le 1/10 de $\mathrm{mm}$, il faut filmer un champ de... $50 \times 50 \mathrm{~mm}^{2}$ !

La photo reste donc irremplaçable lorsqu'on souhaite fixer avec précision une scène étendue $5000 \times 7000$ points environ pour un cliché $24 \times 36 \mathrm{~mm}$ ). Cependant il y a l'attente du développement, peu d'images par minutes... L'espace contre le temps!

De la photo grand champ à la microphotographie

- Stéréophotogrammétrie : voir les volumes de neige déposés.

La répartition des accumulations de neige et leur évolution au cours des variations de conditions nivométéorologiques constituent un élément très important de calage des modèles aussi bien numériques que physiques. Aussi, pour saisir ces dépôts sur une grande surface $(300 \times$ $200 \mathrm{~m}$ ) on a fait appel à la stéréophotogrammétrie terrestre. Depuis un point haut dominant le col du Lac Blanc des clichés sont pris à l'aide d'une caméra stéréophotogrammétrique (Wild P 31). Sur le site, une quinzaine de

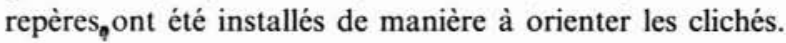
Ces opérations ont été réalisées durant l'été 90 et les premières restitutions effectuées en collaboration avec le laboratoire de photogrammétrie de l'EPFL ont permis de démontrer la faisabilité de la méthode (fig. 5 et 6 , page suiv.). Nous espérons une précision de $15 \mathrm{~cm}$ sur les hauteurs.

Au cours de l'hiver 90-91 on envisage d'effectuer au moins une prise de vue stéréophotogrammétrique par semaine de manière à suivre l'évolution des dépôts de neige.

- Voir la saltation en lumière naturelle : et le blanc devient noir ou rouge.

- Vue de côté : on place une planche noire verticale dans la direction du vent: la caméra ou l'appareil de photo, placé à quelques mètres voit les trajectoires se détacher sur le fond. Soit en filés de longueur proportionnelle à la 


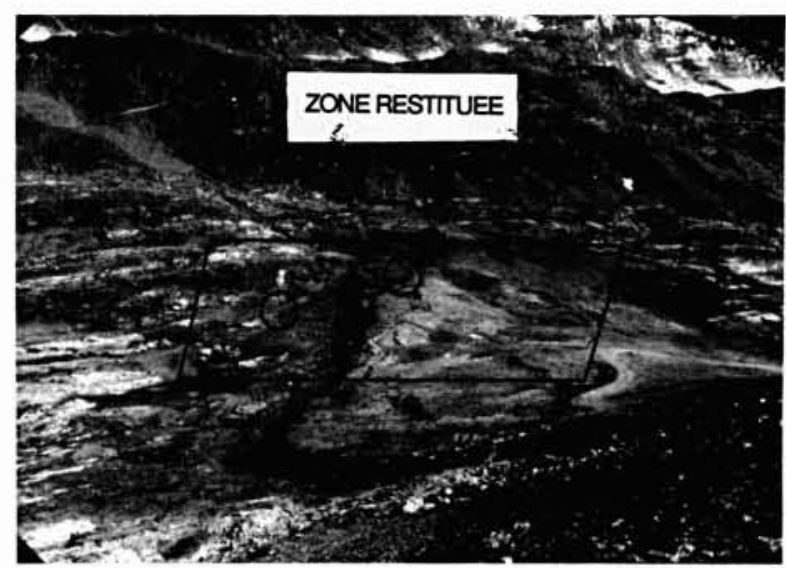

5. Cliché $10 \mathrm{~cm} \times 12 \mathrm{~cm}$ pris avec la caméra stéréophotogrammétrique P31.

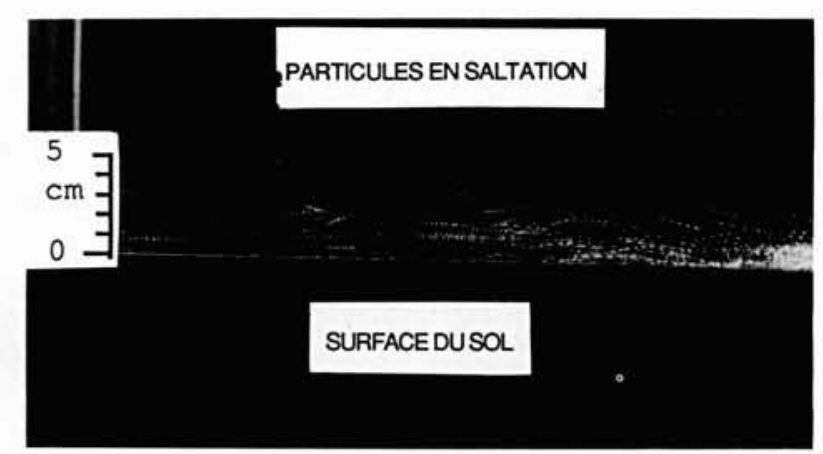

8. Cliché de particules en saltation (soufflerie).

Site du Col du Lac Blanc

Topographie du terrain au $9 / 10 / 90$, maillage de $5 \mathrm{~m}$

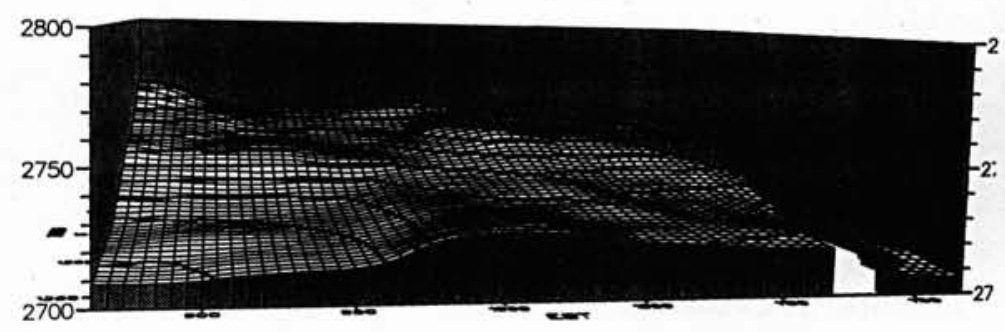

6. Restitution stéréophotogrammétrique de la zone d'étude.

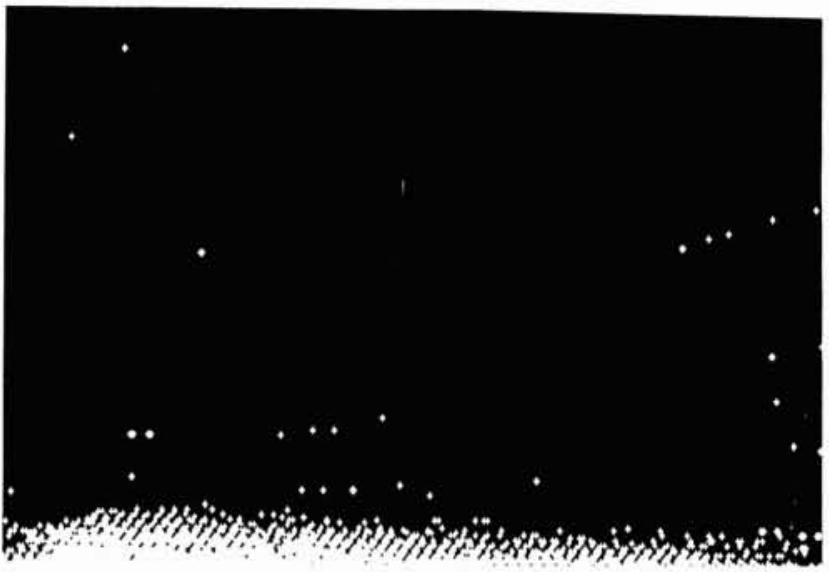

9. Comptage de particules par traitement d'images.
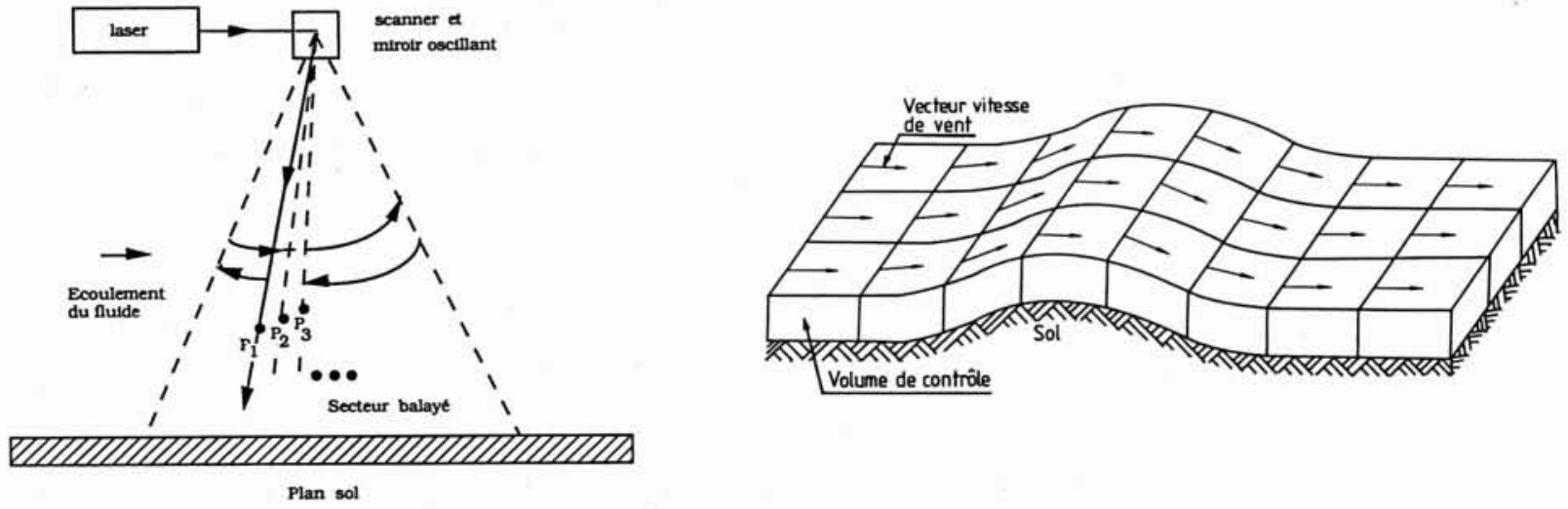

7. Méthode du plan lumineux laser.

10. Principe de calcul du modèle numérique de saltation. 
vitesse (pose longue permettant de voir les trajectoires) soit en nuées de petits points (pose courte permettant des mesures de concentration).

Moyen simple, mais si le vent est très fort, les particules très nombreuses dans l'axe de vision gênent l'observation. - Vue de dessus: on teinte légèrement (pulvérisation d'éosine) une bande de la surface de la neige dans une zone d'érosion : on peut suivre ensuite le déplacement des particules, que l'on repère chaque fois qu'elles s'arrêtent.

- Mesurer des vitesses et des concentrations grâce au laser et à l'ordinateur :

Nous utilisons, tant en soufflerie qu'à l'extérieur, une nappe laser dynamique pour voir le mouvement des particules de neige (trajectoires, vitesse et concentration). Un faisceau laser est réfléchi par un miroir tournant ou oscillant très rapidement ( fig. 7). Le faisceau laser balaye alors l'espace contenu dans une nappe fine triangulaire. Si ce plan est parallèle au déplacement de particules (vent ou axe de la soufflerie) et si les temps sont judicieusement choisis (fréquence de balayage, temps d'obturation caméra) chaque particule en mouvement sera vue comme un pointillé.

En soufflerie le laser a une puissance de $5 \mathrm{~W}$. Il est inutilisable sur site (puissance électrique, refroidissement par eau...). Nous avons donc fait un banc autoorientable dans le vent (girouette) comportant un diode laser $30 \mathrm{~mW}$ dans le proche infrarouge. Ce dispositif compact n'a pas encore été testé.

La scène (fig. 8) est enregistrée sur magnétoscope SVHS ou photographiée, puis l'image est digitalisée (carte Matrox) pour exploitation informatique.

Nous utilisons le logiciel de traitement d'image C-IMA développé pour la mécanique des fluides par le laboratoire de traitement du signal de l'Université de St. Etienne (F). Dans le cadre d'une collaboration, ce logiciel est adapté à nos besoins spécifiques. Il permet de :

- mesurer des vitesses d'éjection et de déposition par appariement de taches dans les zones de faible concentration,

- mesurer des vitesses moyennes des particules par autocorrélation d'image dans les zones à forte concentration, - mesures de concentration par comptage de taches après binarisation et érosion (fig. 9).

Après une première phase de recherche visant à optimiser le traitement à réaliser sur les images, le logiciel constitue actuellement un bon outil d'investigation concernant la dynamique des particules en saltation. Il doit permettre de préciser les termes d'érosion-déposition et de vitesse des particules qui sont utilisées dans le modèle numérique.

- Quelles sont ces particules? cristallographie

Nous réalisons des prélèvements de neige transportée, à différentes hauteurs. Conservés selon un procédé CEN, ils sont descendus pour examen microphotographique à Grenoble.

\subsubsection{Quelques résultats}

Nous disposons désormais d'outils très variés pour acquérir des informations sur le transport de neige par le vent et en particulier la saltation.

Les échanges soufflerie-terrain-modèle s'avèrent déjà très enrichissants : la soufflerie permet de tester certains capteurs (filets, lecteurs de code-barre). Inversement, on compare les profils de congère en soufflerie et sur le terrain, la saltation de la neige avec celle du sable ou de la sciure...

Les premières images en lumière naturelle avec un plan noir ont permis de mettre en évidence le fait que la saltation est accompagnée de phénomènes de reptation, et de tourbillons... L'éosine, comme l'éclairage par laser, laissent supposer que l'hypothèse suivante est vérifiée : une particule en saltation qui se pose n'en éjecte pas immédiatement une autre, mais peut rester immobile quelques secondes, ou se mettre en reptation avant de repartir (aucun rebond ou éjection n'a été enregistré pour l'instant). Ceci montre que le fluide n'a pas une action continue sur la particule mais lui donne une série d'impulsions.

Les films obtenus sont autant d'informations pour la modélisation.

Une fois de plus il est vérifié qu'un équipement simple utilisé dans de bonnes conditions peut apporter de nombreuses informations. Et nous espérons beaucoup de nos outils plus sophistiqués.

\section{Modélisation numérique de transport par saltation}

A partir du travail bibliographique et de nos premières analyses du phénomène nous avons élaboré la maquette d'un modèle de transport de la neige par saltation. Pour cela nous avons divisé le phénomène en deux parties, d'une part le transport proprement dit, et d'autre part l'érosion et le dépôt.

\subsection{La modélisation du transport}

Pour modéliser le transport, l'hypothèse suivante est prise en compte : les grains de neige se déplacent en sautant dans une couche d'air de hauteur limitée. Pour les calculs, cette couche est divisée en volumes élémentaires (ou volumes de contrôles) (fig. 10).

Dans chaque volume, on établit un bilan de la quantité de neige en résolvant l'équation de conservation de la masse :

$$
\frac{\partial C}{\partial t}+\frac{\partial U_{t} C}{\partial x}+\frac{\partial V, C}{\partial y}=\nu\left[\frac{\partial^{2} C}{\partial x^{2}}+\frac{\partial^{2} C}{\partial y^{2}}\right]
$$

Cette équation permet le calcul des flux de neige qui entrent et sortent par les surfaces de chaque volume de contrôle à chaque pas de temps de calcul. 


\subsection{La modélisation de l'érosion et du dépôt}

Afin de modéliser l'érosion et le dépôt, les deux hypothèses suivantes sont proposées :

* A une vitesse de vent donnée, dans un volume élémentaire, la concentration en particules dans l'écoulement augmente en fonction du temps pour atteindre une limite : la concentration de saturation (fig. 11).

* La concentration de saturation augmente avec la vitesse du vent pour atteindre une limite : la concentration limite. De plus, le transport ne peut débuter que lorsque la vitesse du vent est supérieure à un certain seuil : la vitesse seuil.

Ces hypothèses permettent le calcul de la quantité de neige qui est arrachée ou déposée au sol dans chaque volume élémentaire et à chaque instant. Ainsi, la formule suivante a été établie :

$$
Q_{\text {eros }}=\frac{1}{t_{\text {sat }}}\left(C_{\text {sat }}-C\right)
$$

avec :

$Q_{\text {eros }}=$ quantité de neige échangée avec le sol

$t_{\text {sat }}=$ temps nécessaire pour atteindre la saturation

$C_{\text {sat }}=$ concentration de saturation

$=\left(1-\frac{U_{\text {seuil }}}{U_{h}}\right) C_{\text {lim }}$

C = concentration dans le volume de contrôle

$C_{\text {lim }}=$ concentration limite

$U_{\text {seuil }}=$ vitesse seuil

$U_{h} \quad=$ vitesse du vent au-dessus de la couche de saltation

La modélisation de l'ensemble du phénomène de saltation nécessite donc la résolution de l'équation de conservation de la masse suivante :

$$
\frac{\partial C}{\partial t}+\frac{\partial U_{t} C}{\partial x}+\frac{\partial V_{t} C}{\partial y}=\nu\left[\frac{\partial^{2} C}{\partial x^{2}}+\frac{\partial^{2} C}{\partial y^{2}}\right]+Q_{\text {eros }} .
$$

Actuellement le modèle est capable de calculer les concentrations de neige transportée par saltation à condition d'introduire les différents paramètres du transport $\left(U_{v}\right.$,

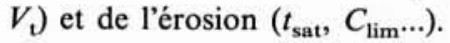

Nous espérons pouvoir déduire tous ces paramètres des vitesses $\left(\mathrm{U}_{\mathrm{h}}\right)$ du vent au niveau du sol à l'aide de lois empiriques établies expérimentalement.

\section{Conclusions}

Au terme de cette première année de recherche, les travaux réalisés portent essentiellement sur les critères de choix et le développement d'une instrumentation adaptée. Dans ce domaine, les objectifs fixés ont été atteints. La saison à venir doit nous permettre d'utiliser cette instrumentation pour atteindre les phénomènes physiques visés et répondre aux questions que l'on se pose.

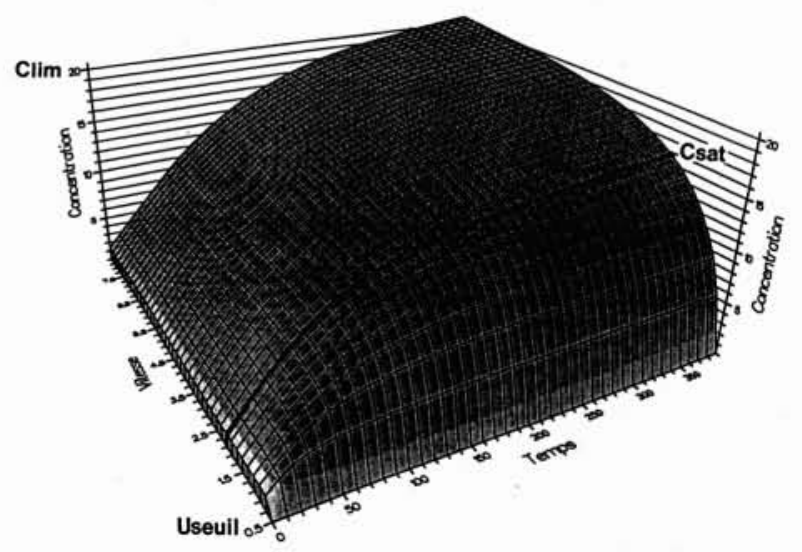

11. Evolution de la concentration de particules dans l'écoulement en fonction de la force du vent et du temps écoulé.

La partie modélisation essentiellement axée sur la simulation numérique du phénomène de saltation constitue une base de travail sur laquelle nous devons porter notre effort : introduction de la topographie et loi de déposition.

La modélisation sur maquette (soufflerie) du transport de neige sur le site expérimental n'a pas encore été étudiée. Cet aspect constitue néanmoins un autre aspect de la recherche que l'on abordera par la suite.

\section{Bibliographie}

BAgnold R.-M., 1941. - The Physics of Blown Sand and Desert Dunes, Chapman and Hall, London.

Castelle T., Hertig J.A., Fallot J.N., 1991. - Protection des routes alpines contre les congères (pour l'Office fédéral des routes). EPFL-Lasen.

FÖHN P.M.B., 1980. - Snow Transport over Mountain Crests, Journal of Glaciology, vol. 26, $\mathrm{n}^{\circ} 94$, pp. 469-480.

Hertig J.-A., 1987. - Protection contre les congères de la route nationale N9 au Simplon, Journal de la Construction, $\mathrm{n}^{\circ} 18$, 15 septembre 1987.

MeISTER R., 1989. - Influence of Strong Winds on Snow Distribution and Avalanche Activity, Annals of Glaciology, 13, pp. 195-201.

OWEN P.R., 1964. - Saltation of Uniform Grains in Air, Journal of Fluid Mech. vol. 20, part 2, pp. 225-242.

RADOK U., 1968. - Deposition and Erosion of Snow by the Wind, CRREL, Research Report, $230 \mathrm{p}$.

SCHMIDT R.A., 1980. - Threshold Wind-speeds and Elastic Impact in Snow Transport, Journal of Glaciology, vol. 26, $n^{\circ} 94$, pp. $453-467$.

TARDU F.S., 1988. - Ecoulement turbulent instationnaire près d'une paroi. Réponse des structures turbulentes (Th. Univ. de Grenoble I).

TAKeUCH M., 1980. - Vertical Profile and Horizontal Increase of Drift-snow Transport, J. of Glaciology, vol. 26, $\mathrm{n}^{\circ} 94$.

Yvergniaux Ph., 1990. - Modélisation de trajectoires de particules solides dans un écoulement turbulent. Thèse "Université Joseph Fournier Grenoble» CEMAGREF Lyon. 